\title{
Origin of the anomalous photovoltaic signal in $\mathrm{Y}-\mathrm{Ba}-\mathrm{Cu}-\mathrm{O}$
}

\author{
H. S. Kwok, J. P. Zheng, and S. Y. Dong \\ Institute on Superconductivity, State University of New York at Buffalo, Bonner Hall, Amherst, New York 14260 \\ (Received 19 October 1990)
}

\begin{abstract}
The temperature and polarization dependence, and anisotropy of the laser-generated voltage response in $\mathrm{Y}-\mathrm{Ba}-\mathrm{Cu}-\mathrm{O}$ under no-current-bias conditions were examined. It was found that the photogenerated current flowed predominantly along the $c$ axis of the $\mathrm{Y}-\mathrm{Ba}-\mathrm{Cu}-\mathrm{O}$ crystal, and was laser-polarization independent. The strong temperature dependence observed indicated that the signal was related to the $240-\mathrm{K}$ structural transformation which was also responsible for other anomalies in the early days of $\mathrm{Y}-\mathrm{Ba}-\mathrm{Cu}-\mathrm{O}$ research. The anomalous photovoltaic response is due to an asymmetry along the $c$ axis of the $\mathrm{Y}-\mathrm{Ba}-\mathrm{Cu}-\mathrm{O}$ orthorhombic crystalline structure.
\end{abstract}

Recently, Chang, Kleinhammes, Moulton, and Testardi reported the observation of laser-induced transient voltages in thin films of $\mathrm{Y}-\mathrm{Ba}-\mathrm{Cu}-\mathrm{O}$ (Ref. 1). They excluded thermal effects as the possible cause of these signals. Tate, Johnson, Chang, and Hilinski used picosecond laser pulses and observed similar voltages at similar laser fluences. ${ }^{2}$ Additionally, they reported that the signal polarity was reversed when the laser was incident on the opposite side of the film. Scott explained these observations based on the third-rank photovoltaic tensor. ${ }^{3}$

In this paper, we report some careful measurements of the photovoltaic effect using well characterized and oriented thin films. Our results indicate that: (i) The signal is extremely anisotropic, with the photoinduced current flowing along the $c$ axis of the $\mathrm{Y}-\mathrm{Ba}-\mathrm{Cu}-\mathrm{O}$ lattice only. (ii) The signal is laser-polarization independent. (iii) The effect is strongly temperature dependent, showing two temperature onset thresholds. (iv) The laserinduced voltage or current occur not only in the transient mode; a dc voltage can be maintained by using a cw laser source. (v) The signal is present mainly in the orthorhombic phase of $\mathrm{Y}-\mathrm{Ba}-\mathrm{Cu}-\mathrm{O}$, and not in the tetragonal phase. We interpret the result as due to the $240-\mathrm{K}$ structural transformation of the orthorhombic crystal lattice from Pmmm to Pmm2 which has an asymmetric $c$ axis.

The thin films used in these experiments were laser deposited on $\mathrm{SrTiO}_{3}(110)$ substrates. The lattice-matching conditions allow the growth of grains with the $c$ axis at $45^{\circ}$ to the surface, and also parallel to the surface. By using $x$-ray-texture analysis it was confirmed that all the

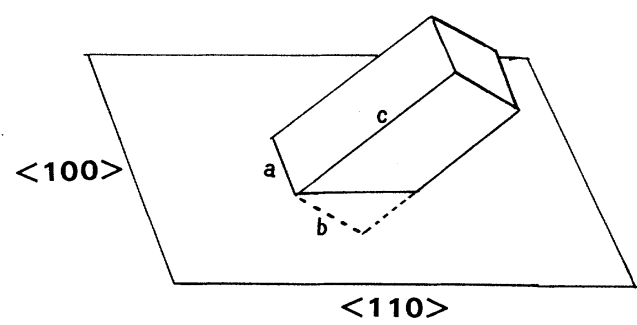

FIG. 1. Position of the $\mathrm{Y}-\mathrm{Ba}-\mathrm{Cu}-\mathrm{O}$ unit cell relative to the substrate surface. The $c$ axis can tilt in either direction. grains had their $a$-axes parallel to the $\langle 100\rangle$ direction of the substrate, and the $c$ axes at $45^{\circ}$ to the $\langle 110\rangle$ substrate axis as shown in Fig. $1 .^{4}$ There was a negligible amount of grains in the other directions. The $T_{c}$ of these highquality films were $88 \mathrm{~K}$.

The following experiment was then performed. Many pairs of electrodes were bonded to the film in a circular fashion as shown in Fig. 2. A chopped He-Ne laser irradiated the center of the film. A lock-in amplifier was used to detect the signal as a function of the measurement angle $\Phi$, without changing any laser parameters such as intensity and position. The angle $\Phi$ is defined as the angle between the [100] direction of the substrate and the line connecting the two measuring electrodes. Figure 3 shows the results. It can be seen that there is a very strong dependence of the photovoltaic signal on the angle $\Phi$. The signal shows a strong peak at $\Phi=90^{\circ}$ which is in the same direction as the $\mathrm{Y}-\mathrm{Ba}-\mathrm{Cu}-\mathrm{O} c$ axis. The uncertainty in $\Phi$ is $\pm 5^{\circ}$ due to the size of the bonding pads. Nevertheless this result indicates unequivocally that when light is incident on the film, the photogenerated current flows only along the $c$ axis of the $\mathrm{Y}-\mathrm{Ba}-\mathrm{Cu}-\mathrm{O}$ crystal.

The fact that the photogenerated current flows along the $c$ axis was confirmed in many samples. For $\mathrm{Y}-\mathrm{Ba}$ $\mathrm{Cu}-\mathrm{O}$ films with the $c$-axis perpendicular to the substrate surface, such as on $\mathrm{MgO}(100)$ or $\mathrm{SrTiO}_{3}(100)$, it was found that the magnitude of the signal was strongly dependent on the tilt of the $c$ axis. It is a well-known fact

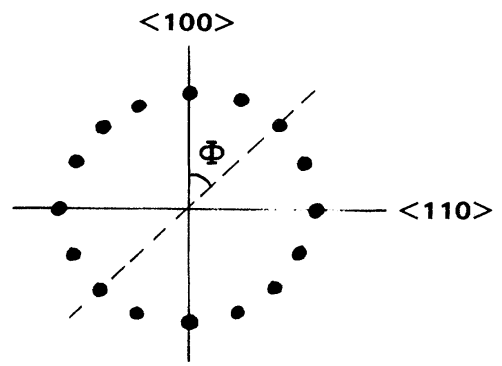

FIG. 2. Position of the measuring electrodes on the film surface. The laser irradiates the center $1 \mathrm{~mm}^{2}$. Electrode spacing $\sim 8 \mathrm{~mm}$. 


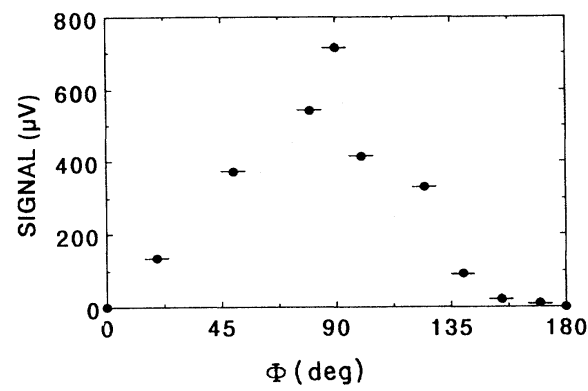

FIG. 3. Photovoltaic signal as a function of the measuring angle $\Phi$.

that commercial (100) surface substrates do not exactly have the [100] axis perpendicular to the surface. It is usually tilted by $0.1^{\circ}-3^{\circ}$. Therefore, the $\mathrm{Y}-\mathrm{Ba}-\mathrm{Cu}-\mathrm{O} c$ axis deposited on such films is also slightly tilted. We found that films with a tilt angle of $1.5^{\circ}$ produced photovoltaic signals almost 20 times larger than those with only a $0.3^{\circ}$ tilt. Films with the $c$ axis exactly normal to the surface produced negligible signals. For the $45^{\circ}$ films used in most of the present experiment, there is an $\sim 1^{\circ}$ tilt of the [110] substrate axis from the surface normal. This tilt is probably responsible for the observed signal, since the grains can have the $c$-axis pointing in either direction of the substrate $\langle 110\rangle$ axis.

By using a $20-\mathrm{mW} \mathrm{cw}$ He-Ne laser, a de signal of 0.7 $\mathrm{mV}$ could be obtained with $10^{5}$ amplification. Thus this laser-induced effect is not only a transient phenomenon, it is a true photovoltaic effect. By rotating the polarization of the laser, it was found that the signal did not change, indicating that there was no polarization dependence. Thus the explanation proposed in Ref. 3 cannot be valid. Additionally, we did not observe any reversal of the signal voltage upon shining the laser from the back of the films, contrary to the results reported in Ref. 2. The independence of the signal on the propagation vector of the light is consistent with the lack of polarization dependence. It rules out any tensorial effects in the photovoltaic signal such as in piezoelectricity.

Using the chopped He-Ne laser, the heating of the film was negligible. It was then possible to study the temperature dependence of the signal. Figure 4 shows the experimental results in both linear and log scales. It can be seen that there is a strong temperature dependence. There are two apparent thresholds at 90 and $\sim 200 \mathrm{~K}$. This result is contradictory to those reported in Refs. 1 and 2 where no temperature dependence was observed. We believe that the discrepancy is due to the use of intense pulsed lasers in those cases leading to large temperature increases. We indeed confirmed this point by using a pulsed Nd:YAG (where YAG denotes yttrium aluminum garnet) laser as the excitation source. In that case, there was only a slight temperature dependence in the signal.

Since the signal can exist in a dc mode and is strongly temperature dependent, it cannot be thermal in origin. The major threshold for the photovoltaic signal at $\sim 200$ $\mathrm{K}$ is reminiscent of the $240-\mathrm{K}$ anomaly observed during the early days of $\mathrm{Y}-\mathrm{Ba}-\mathrm{Cu}-\mathrm{O}$ research. It was seen in $\mathrm{Ra}-$ man scattering, ultrasound attentuation, and sound veloci-
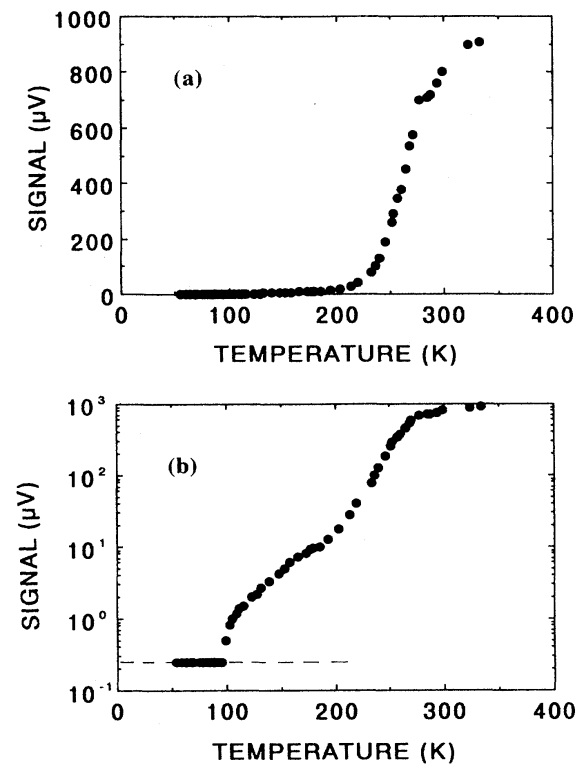

FIG. 4. Temperature dependence of the photovoltaic signal. The horizontal line is the noise limit; (a) linear scale showing the major $(\sim 200 \mathrm{~K})$ threshold; (b) log scale showing the lower $(90 \mathrm{~K})$ threshold.

ty measurements ${ }^{5}$ and was confirmed to be related to a Pmmm to Pmm2 structural transformation of the orthorhombic lattice. Above this temperature, the crystal structure is asymmetric along the $c$ axis. This asymmetry is crucial in explaining the origin of the photovoltaic signal. ${ }^{1,3}$ The situation is actually similar to the photogalvanic effect (PGE) for material without a center of symmetry. ${ }^{6}$ In Ref. 6 , it was pointed out that the photogenerated voltage was present even in gases and liquids possessing optical activity. For PGE, it is not necessary to have a built-in electric field which is generally the case for ordinary photovoltaic effects in a $p$ - $n$ junction.

With the above data, a plausible argument can be given on the origin of the photovoltaic signal. In $\mathrm{Y}-\mathrm{Ba}-\mathrm{Cu}-\mathrm{O}$, the mobile charge carriers are confined to the $\mathrm{CuO}_{2}$ planes. Interplane transport occurs only when this confinement is violated, such as by strong thermal fluctuations. ${ }^{7}$ In many models, current transport along the $c$ axis can be viewed as a tunneling or hopping process between these planes. ${ }^{8}$ When the light irradiates the sample, the carriers are excited and can hop or tunnel between planes with higher mobility. The asymmetry of the lattice along the $c$ axis should induce an asymmetric tunneling or hopping of the excited carriers. If the carriers move in a preferred direction, a current will be generated. A more quantitative model calculation on this process can probably be presented in the future with further theoretical insight. At present we have no explanation for the weak signal above $90 \mathrm{~K}$. It is perhaps related to the superconducting transition or may be due to thermal fluctuation effects. We do not believe that the asymmetry along the $c$ axis in $\mathrm{Y}-\mathrm{Ba}-\mathrm{Cu}-\mathrm{O}$ generates an electric field as in ordinary ferroelectric crystals. Such fields, if present, will be screened by the mobile holes resulting in an asymmetric equilibri- 
um charge distribution. This should not be the case.

Since the sample is polycrystalline, it is uncertain why there is an overall preferred direction for the flow of the photogenerated current. Suppose the asymmetry occurs by displacement of the cations in one direction along the [001] axis, then arguably, the direction of this shifting should not be coordinated between the grains, i.e., there should be an equal number of grains with cations shifting in one direction as in the opposite direction, Hence there should be no net current flow. Perhaps there is a preferred direction of this supposed cation displacement because of the presence of the substrate. To avoid such uncertainties in the interpretation, the present experiment should be performed in an untwinned single crystal.

Based on the magnitude of the photovoltaic signal, the displacement of the cations can be estimated to be extremely small. This may or may not be observable by $x-$ ray diffraction. Such $\mathrm{x}$-ray measurements are meaningful and should be performed not only on $\mathrm{Y}-\mathrm{Ba}-\mathrm{Cu}-\mathrm{O}$, but on other oxide superconductors as well. It may well turn out that this small asymmetry is related to the superconducting mechanism in these materials.
By performing the photovoltaic measurement in the vacuum deposition chamber, it is possible to examine the effect of different crystalline phases on the signal. We can heat up the sample in either an oxygen or vacuum environment to obtain the orthorhombic or tetragonal phases. ${ }^{9}$ It was found that the signal was at least 30 times higher for the film in the oxygen rich phase. This basically confirms that the signal is related to the crystalline transformation in the orthorhombic phase. It also implies that the tetragonal phase does not have this interesting structural transformation.

The photovoltaic response is intimately related to the anisotropy of the lattice and can be used to study the crystal structures of these perovskites. It may be used to study the normal-state characteristics of the high- $T_{c}$ materials. However, this effect is quite different from the normal photoresponse under current bias. The latter is generally much stronger and both thermal and nonthermal responses have been demonstrated. ${ }^{10}$

This research was supported by National Science Foundation under Grant No. ECS9006847.
${ }^{1}$ C. L. Chang, A. Kleinhammes, W. G. Moulton, and L. R. Testardi, Phys. Rev. B 41, 11564 (1990).

${ }^{2}$ K. L. Tate, R. D. Johnson, C. L. Chang, and E. F. Hilinski, J. Appl. Phys. 67, 4375 (1990).

${ }^{3}$ J. F. Scott, Appl. Phys. Lett. 56, 1914 (1990).

${ }^{4}$ S. Y. Dong, J. P. Zheng, and H. S. Kwok (unpublished).

${ }^{5}$ M. S. Zhang, C. Qiang, D. K. Sun, R. F. Ji, Z. K. Qin, Y. Zheng, and J. F. Scott, Solid State Commun. 65, 487 (1988).

${ }^{6}$ V. Belinitcher and B. Sturman, Usp Fiz. Nauk. 130, 415
(1980) [Sov. Phys. Usp. 23, 199 (1980)].

${ }^{7}$ D. A. Brawner, Z. Z. Wang, and N. P. Ong, Phys. Rev. B 40, 9329 (1989).

${ }^{8}$ P. W. Anderson and Z. Zou, Phys. Rev. Lett. 60, 132 (1988).

${ }^{9}$ Q. Y. Ying, H. S. Kim, D. T. Shaw, and H. S. Kwok, Appl. Phys. Lett. 55, 1041 (1989).

${ }^{10}$ H. S. Kwok, J. P. Zheng, and R. Rao, Appl. Phys. Lett. 54, 2473 (1989). 